\title{
MÉTODOS, FORMAS, PROCEDIMIENTOS Y SISTEMAS DE ENSEÑANZA EN UNA CONCEPCIÓN DE LA PEDAGOGÍA COMO ARTE SEGÚN JOSÉ M. ZAMORA
}

\section{Methods, forms, procedures and education systems on a conception of teaching as art by José M. Zamora}

\author{
Martha Lucía Orozco Gómez \\ Universidad de Burgos. Facultad de Educación \\ Correo-e: mlorozco@ubu.es \\ Recepción: 22 de febrero de 2014 \\ Envío a informantes: 29 de julio de 2014 \\ Fecha de aceptación definitiva: 2 de febrero de 2015 \\ Biblid. [0214-3402 (2015) (II época) n.o 2I; 165-176]
}

Resumen: En el presente artículo se recrean categorías centrales del ámbito pedagógico y su problematización en elementos como los métodos, las formas, los procedimientos, los sistemas y las leyes. Todo ello, como parte de la recuperación de la memoria pedagógica por medio de la discursividad que en relación a este campo del saber se realiza en los desarrollos de José M. Zamora, como una reforma que inspiró las prácticas pedagógicas de un tramo de la Historia de la Educación, los años 2o, e inspirada especialmente por la primera misión pedagógica alemana.

En el Manual de Pedagogía de Zamora, se vislumbran las posibilidades y visiones de un mundo esperanzador desde la Educación. Unos componentes que llegan al tiempo presente para ser valorados. El autor considerado divide los métodos educativos en tres: físicos, intelectual y moral; las formas pedagógicas, las que clasifica en cuatro: interrogativa, expositiva, heurística y narrativa; los procedimientos pedagógicos los divide en diez; los sistemas pedagógicos en tres: mutuo o recíproco, conferencias y discusión; las leyes pedagógicas: física, intelectual y moral. Todos elementos importantes en el análisis pedagógico contemporáneo.

Palabras Clave: métodos; formas; procedimientos; sistemas; leyes; pedagogía.

Aвstract: In this article, core pedagogic categories and its problematic elements such as: methods, forms, procedures, systems and laws are recreated. All this, as part of the pedagogical memory recovery through the discourse, in relation to this field of knowledge, that is done in the developments of José M. Zamora, as a reform that 
inspired the pedagogical practices during a period of the History of Education, the 2os, and especially inspired by the first German pedagogical mission.

In the Manual of Pedagogy of Zamora, possibilities and visions from a hopeful World from the Education are seen. Some components arrive at the present time to be evaluated. The author distinguishes into three educational methods: physical, intellectual and moral; Pedagogical forms are classified into four: interrogative, expository, heuristics and narrative. The pedagogical procedures are divided in ten, whereas the educational systems in three: mutual or reciprocal, conferences and discussion. Finally, the pedagogical laws are divided in: physical, intellectual and moral. All of them are important elements in the contemporary pedagogical analysis.

KEY WORDs: methods; forms; procedures; systems; law; pedagogy.

\section{Introducción}

La pedagogía tiene por objeto principal el estudio del desarrollo humano, investigando su punto de partida, sus leyes y su fin, y por esto se le llama ciencia, pero considerada como arte podemos decir que enseña los procedimientos, las formas y los métodos más adecuados para educar al hombre según aquellas leyes.

José M. Zamora

$\mathrm{E}$ N i924, el maestro José M. Zamora, alumno de Ernesto Hotschink², publicó en Bogotá-Colombia el libro Pedagogía, texto que recogía las conferencias arregladas como cursos de Historia, Metodología General, Especial y Legislación Pública, dictados en la Normal de Varones de Tunja y en el colegio de la Merced en Bogotá. En su momento, Zamora se preocupó por abordar el estudio de la Pedagogía desde una perspectiva pragmática, fiel a los principios puestos en vigencia por los pedagogos alemanes, de ahí el que tome como aspecto central los métodos de enseñanza e intente verlos como leyes regulares que pueden cumplirse y que en esencia es lo que le da a la Pedagogía -en su concepción- su carácter científico.

\section{Los métodos de enseñanza en educación y la pedagogía}

Zamora (1924: I03) consideraba los métodos de enseñanza como una parte fundamental de la Pedagogía ya que de éstos dependían los logros que se obtuvieran en la educación del hombre, partiendo de considerar el método como «la ordenada distribución de los actos encaminados a la consecución de un fin».

Así, al ocuparse sólo de los procesos de enseñanza, busca una definición más apropiada a tal fin, e intenta centrar más su atención en este aspecto, llamándolo Método Pedagógico al que define como «la ordenada distribución de los actos del educador, encaminados a conseguir la educación del hombre» (Zamora, I924: 103), que debe

Pedagogo diplomado de grado superior discípulo del profesor alemán Ernesto Hotschick. Conferencias dictadas en la Normal de Varones de Tunja (Colombia); en el Colegio de La Merced, en Bogotá; en el de la Provincia, en Madrid y adoptadas en varios establecimientos. 1924-1928.

Eminente pedagogo que había arribado a Colombia en la primera misión pedagógica procedente de Alemania. 
MÉTODOS, FORMAS, PROCEDIMIENTOS Y SISTEMAS DE ENSEÑANZA EN UNA CONCEPCIÓN...

conllevar la formación de un ser integral abarcando sus condiciones físicas, intelectuales y morales.

Según el autor, cada uno de estos aspectos que son indispensables para un desarrollo armónico del hombre necesita de un método especial y particular de enseñanza, por lo cual considera tres clases de métodos: aquellos que se relacionan con aspectos materiales, los que ven al ser intelectual y los que sirven a la formación moral.

\section{I.I. Método físico}

Los métodos relacionados con la parte material o física se dividen, según Zamora, en tres conocidos en la época, denominados: natural racional, contranatural y contrarracional.

El método natural racional busca educar la parte física del hombre teniendo en cuenta su naturaleza "y sin apartarse de la razón que debe guiar al educador», considera al individuo como un ser con capacidades y limitaciones propias. Como ejemplos el autor nos señala cómo para un niño de ocho años que recibe una clase de educación física, su maestro debe saber cuáles son las facultades que para su edad debe tener y de esta manera programar los ejercicios correspondientes o una madre que llevada por la razón da a su hijo la cantidad de leche que necesita sin excederse. Aquí debe entenderse que la aplicación de este método está relacionada únicamente con la lógica de la razón, teniendo en cuenta las necesidades y capacidades de cada individuo (Zamora, I924: I03).

Los métodos contranatural y contrarracional contradicen lo anterior ya que, como sus nombres lo indican, y así lo sugiere el autor, van en contra de las facultades naturales del niño y en contra de la razón del profesor. Esto ocurre cuando se obliga al niño a realizar actividades superiores a sus fuerzas, las que no logrará cumplir por más deseo que tenga de hacerlo y agradar a su profesor. Tal método empleado entonces es inaceptable, haciendo justicia a su nombre y llevando de paso al fracaso del proceso educativo. Además, «como la educación debe ser gradual y progresiva, se requiere tomar un punto de partida, concebir un plan y desarrollarlo dentro de estos límites, de manera que pueda palparse el fruto» (Zamora, I924: I04).

Para el autor, los métodos tienen unos auxiliares tales como: las formas, los procedimientos y los sistemas pedagógicos, resaltando el beneficio que el buen uso y el correcto conocimiento de estos métodos pueden dar al aplicarlos en la enseñanza, pero insiste en que es una tarea delicada donde el maestro debe prepararse bien y tener un gran conocimiento de la materia siendo a la vez celoso en el desempeño de sus obligaciones.

\section{I.2. Método intelectual y el ser}

Por otra parte, en la lectura del texto de Zamora encontramos la educación intelectual, que es la encargada de mirar al ser intelectual y la que divide en tres: el método de evidencia previa, el método de evidencia posterior inmediata y el método de evidencia posterior mediata.

El método de evidencia previa plantea lo que hoy es uno de los elementos fundamentales de la corriente constructivista: darle una gran importancia a los conocimientos 
previos del niño ya sean adquiridos en el hogar, la familia, de la madre, de sus juguetes o de todos aquellos objetos que vayan siendo de su conocimiento.

Al respecto, Flórez (1994) señala:

La enseñanza constructivista considera que el aprendizaje humano es siempre una construcción interior, aun en el caso de que el educador acuda a una exposición magistral, pues ésta no puede ser significativa si sus conceptos no encajan ni se ensartan en los conceptos previos de los alumnos. [...] la idea de enseñanza como transmisión mecánica de información de un sujeto activo a otro pasivo es imposible hasta en la enseñanza más tradicionalista, porque de hecho nunca la cabeza del alumno está vacía. Aunque el maestro no quiera, el procesamiento interior de cualquier mensaje que les llega a los alumnos es inevitable.

Para Zamora (I924: I05), «por este método se va de lo conocido a lo desconocido, de lo inmediato a lo mediato, de lo simple a lo compuesto, de lo concreto a lo abstracto $y$, por tanto, nos ha parecido el más adecuado para la enseñanza primaria», pues considera que este método ayuda a una edificación sobre bases sólidas, y da como ejemplo una clase en la que se toma como punto de partida un elemento conocido por el niño «puesto que es sabido que lo que hiere la vista hiere la imaginación y herida la imaginación se sugestiona la voluntad».

El segundo método, de evidencia posterior inmediata, es contrario al anterior en el sentido de que aquí el maestro no tiene en cuenta los conocimientos del niño, sino que brinda sus propios conocimientos a través de una exposición clara y concreta que le permita al estudiante comprender y asimilar de tal forma que pueda repetirlo con sus propias palabras y le sirva en el trato con la vida, aclarando a la vez que no es necesaria la memorización esclavizante. En este particular, traemos a colación aquel precepto de la pedagogía propuesto por Séneca: «Aprendemos para la vida y no para la escuela», es decir, centra su atención en la adecuada comunicación de los conocimientos para la posterior vida de relación del individuo.

De acuerdo con el autor, este método es adecuado para aquellos niños que en la sección elemental han sido preparados en la enseñanza objetiva, ya que sus facultades se hallan en las mejores condiciones y aclara ejemplificando:

Se pretende enseñar al niño que sílaba es una o más letras que se pronuncian en una sola emisión de la voz, para lo cual se aprende primero la definición; se toman luego palabras que nombren objetos que les sean conocidos y las haremos pronunciar dividiéndolas, surgiendo de aquí las sílabas; luego estableceremos la comparación por el número de letras que contengan, y aunque el niño puede no dar el nombre de emisión sino cualquier otro, nos arreglaremos de manera que establezcamos los cambios indispensables para obtener la definición correcta. Una buena inducción hace que el resultado sea satisfactorio. Aquí hemos ido de lo general a lo particular, del todo a las partes (Zamora, 1924: 106).

El tercer método planteado, de evidencia posterior mediata, da crédito al aprendizaje memorístico y de él se espera que con el tiempo el estudiante llegue a comprender lo aprendido. Este método que solo tiene en cuenta el desarrollo de la memoria fue empleado por Lancaster y probó el «atrofiamiento» del entendimiento, según el autor, lo que llevó a su fracaso en un corto tiempo. Zamora consideraba este método perjudicial sobre todo cuando se trata de niños y piensa que lo más beneficioso en 
MÉTODOS, FORMAS, PROCEDIMIENTOS Y SISTEMAS DE ENSEÑANZA EN UNA CONCEPCIÓN... MARTHA LUCÍA OROZCO GÓMEZ

cualquier proceso cognitivo y de desarrollo para las facultades mentales se encuentra en el desarrollo del entendimiento como base de un buen cultivo de la memoria.

Para aclarar aún más sobre este método el autor señala cómo un maestro que desea enseñar a sus estudiantes que los ángulos de todo triángulo miden juntos $180^{\circ}$, exponiendo simplemente la definición, de pura memoria sólo consigue que los niños la repitan sin equivocación pero sin comprenderla, pues el docente sólo ha realizado una exposición teórica sin facilitar el transportador y lo que es peor aún sin siquiera conocer el instrumento y su nomenclatura. Con el paso de los años los niños se convierten en hombres y ocasionalmente tropiezan con los instrumentos de geometría que alguno les describe; es entonces cuando el hombre, formado ya, se da cuenta de la definición que de niño aprendió en la escuela, lo que prueba que este método no es provechoso ni puede adoptarse. El propio Zamora resume: «El que ha aprendido de memoria, nada ha aprendido y el que aprende como loro, loro ignorante se queda» (Zamora, 1924: 107).

\section{I.3. Método moral y el ser}

De otro lado, de las partes más importantes del ser humano, su educación moral, según Zamora, es aquella que deberá guiar todos sus actos en sociedad, en su familia y consigo mismo, es decir, esta educación tiene como fin el perfeccionamiento del espíritu y de ahí que se refleje un vínculo muy cercano con la psicología que además es una parte de la filosofía que trata del alma, sus facultades y operaciones.

Tratándose de la parte moral, el autor resalta dos grandes métodos: el ideológico y el pedagógico. Sin embargo, señala que hay quienes admiten un tercero llamado de gracia, que hace referencia a lo divino, a algo sobrenatural de lo cual el individuo es un instrumento.

De esta manera, el método ideológico «es aquel que hace que el hombre se dirija por su propia idea» (Zamora, I924: I08), sin embargo, esto no garantiza que se pueda lograr un mismo fin común a todos aquellos que lo utilicen, pues el hombre por naturaleza goza de diversidad de caracteres, de inclinaciones y de temperamentos. En otras palabras, este método acude a la formación más noble del hombre, teniendo presente su propia idea del bien y del mal, acudiendo a su propia razón y al llamado de su conciencia para inclinarse por el uno o el otro.

Seguir el mal -o lo que llama el autor el libre albedrío- o elegir el bien es producto de la primera enseñanza que sin duda alguna es dada en el hogar, lo que hace responsables a los padres de infundir en sus hijos una buena educación moral que permita recoger unos buenos frutos, lo que se lograría mediante el ejemplo dado, de lo contrario deberá asumirse el resultado. Ahora bien, es claro que la responsabilidad del maestro no es poca cuando se trata de la educación moral de los niños con base en la consideración de que la escuela es la continuidad del hogar y los maestros los representantes de los padres, especialmente en la etapa inicial del proceso educativo. Así, el maestro tiene la responsabilidad, según Zamora (1924), de reforzar las buenas disposiciones morales estimulando la virtud, la dignidad, exhortando al bien y educando la voluntad, sobre todo si no se tiene el buen ejemplo de los padres en casa.

Agrega además que el maestro puede -a través de los consejos, las buenas lecturas, los relatos de personas que han sobresalido por ser íntegras y de sanas costumbresllegar más fácilmente a moldear el alma del niño. 
Seguidamente, hace referencia al método pedagógico como «aquel en el cual se va a la práctica del bien mediante las enseñanzas y los auxilios directos del maestro, y esas enseñanzas deben ser asesoradas del ejemplo, como que este es insentivo (sic) poderoso que hace inclinar la voluntad» (Zamora, I924: I09). Es importante resaltar aquí la importancia que tiene en cualquier etapa de la educación que la teoría que enseñamos, a través de la cual hacemos partícipes a los estudiantes de las discusiones que sobre moral se han dado, sea reforzada realmente con el ejemplo, puesto que a través de muchos medios el niño está recibiendo información que de una u otra manera afecta a su comportamiento moral, sus creencias y convicciones frente a la vida. Finalmente, la educación moral busca «enseñar el bien y hacer que se exterioricen los buenos sentimientos...» (Zamora, I924: IIO), contribuyendo esto a formar una sociedad justa y digna que prepare más hombres para la paz y menos para la guerra.

Gráfico original en el que se sintetizan los métodos de educación

(ZAMORA, I924: IIO)

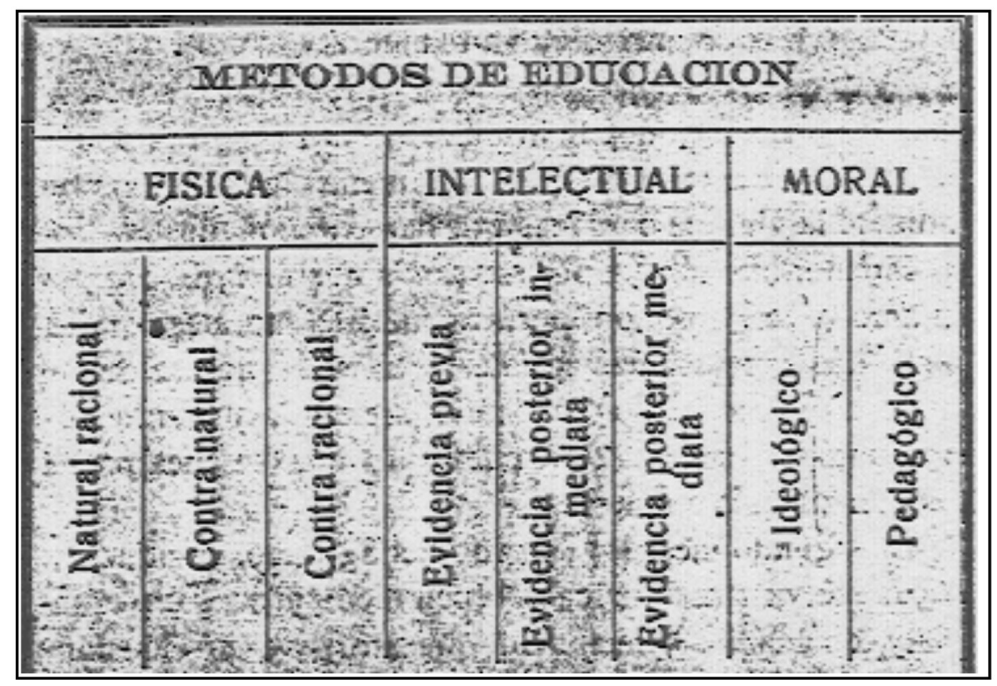

\section{Las formas pedagógicas}

Una vez que el autor nos describe detalladamente los métodos de educación pasa a abordar las formas pedagógicas, las que clasifica en cuatro: interrogativa, expositiva, heurística y narrativa, asumiendo la definición de forma como «la disposición que se adopte para dar la enseñanza...» (Zamora, I924: III).

La forma pedagógica interrogativa, la define como «aquella que tiene por base la pregunta». También, ha sido llamada de otras maneras como: socrática ${ }^{3}$, dialogística,

Conocida con este nombre gracias a que este método fue utilizado por el filósofo griego Sócrates, quien enseñaba a sus discípulos la verdad de esta manera y en especial a su gran amigo Aristodemo. 
gracias al diálogo que obligatoriamente se establece entre el interlocutor y el interpelado mediante la realización de preguntas y sus respectivas respuestas, y catequística, por ser ésta la forma más empleada en la enseñanza del catecismo. Y considera que a través de esta forma se adquieren resultados prácticos, ya que ayuda a mantener la atención, a desarrollar el interés por la investigación y a la exposición de sus propias ideas, según lo que desea descubrir.

Por lo anterior, Zamora (1924) considera que la forma interrogativa está apoyada fuertemente en los procedimientos inductivo-deductivos y la forma heurística, lo que no quiere decir que deba tratarse de cualquier manera. De hecho, se reconoce que la dificultad de muchos educandos en los procesos evaluativos es la falta de comprensión de las preguntas que le son formuladas ya que algunos profesores se han especializado en realizar preguntas oscuras, capciosas (o mal intencionadas) y largas, en lugar de elaborar preguntas claras, cortas y concisas. De igual manera, Zamora (1924) considera que el educando, al responder, debe hacerlo incluyendo la pregunta y además en voz alta que refleje seguridad e interés, lo que a la vez le ayuda a desarrollar la vocalización y los pulmones.

La segunda forma pedagógica, conocida como expositiva, se basa «en la presentación o exposición de los puntos que lleva preparados el maestro para enseñar» ( $\mathrm{Za}$ mora, I924: II2). Esta forma pedagógica es el soporte principal del método de evidencia posterior inmediata que se describiera anteriormente y que para la enseñanza de algunas materias podría ser adecuado siempre y cuando el maestro, con su ingenio, logre mantener la atención de los educandos, pues una exposición larga y poco clara puede hacer que el niño, que fácilmente se distrae, pierda la secuencia de las ideas y finalmente se pierda el tiempo de ambos actores en este proceso. Si esta forma se utiliza con esmero y buen tino puede lograrse un aprendizaje inmediato en el niño, puesto que una buena narración también puede lograr efectos positivos en el auditorio. A principios de siglo se consideraba que esta forma había sido bien empleada si el educando era capaz de realizar su propia conclusión en lo cívico y lo moral que era el ritual de la época. En algunos casos también se le llama narrativa.

La tercera forma, denominada eurística (sic), es un gran auxiliar del método de evidencia previa, siendo a la vez afín con el de evidencia posterior inmediata y puede comprenderse como «aquella en que se descubre alguna verdad» ${ }^{4}$.

Para Zamora, la aplicación de esta forma es peculiar de un buen «genio pedagógico» y «enlazar la enseñanza de una manera gradual y progresiva, equivale a colocar los eslabones de una cadena sabiamente...», en este propósito es fundamental el apoyo de la forma interrogativa. Las ventajas que se obtienen por esta forma son muchas ya que a través de él se despierta el interés y se fortalece el amor propio del educando, pues, él cree que el hallar una respuesta oportuna o descubrir una verdad es el producto de su ingenio, capacidad mental y dedicación a lo que hace (Zamora, I924: II3).

Finalmente, se aborda la última forma pedagógica, denominada narrativa, que es «aquella de que se vale el educador para relatar pasajes de historia...», esta forma,

4 Llamada de esta manera porque viene de la palabra Eureka, voz de que se valió Arquímedes, el gran matemático, para manifestar su satisfacción al descubrir la ley de física sobre el peso de los cuerpos sumergidos en los líquidos. Siracusa, población importante de Italia, fue testigo de la alegría de Arquímedes, quien, al salir del baño, recorría las calles gritando eureka! eureka!, que quiere decir: descubrí, hallé, encontré. Posiblemente por el propio origen de la palabra en muchos documentos antiguos se encuentra escrito eurística en vez de heurística como se escribe actualmente, tal y como es el caso del Manual de Zamora. 
de cierta manera, se encarna en algunas ocasiones en la expositiva y tiene afinidades con la interrogativa, dando como resultado una forma mixta que Zamora denomina narrativo-interrogativa, además, indica que si esta forma es bien utilizada se podrá saber de viva voz del educando los conocimientos que haya asimilado y añade que la enseñanza manejada de esta manera toma el nombre de «procedimiento oral» (Zamora, I924: II3).

Es importante resaltar que esta forma debe ser bien manejada de la misma manera que la forma expositiva, ya que los niños de primaria se distraen con facilidad y relatos muy largos y carentes de interés sólo lograrán que el educando se distraiga más rápido. Debe motivarse de vez en cuando con la pregunta, que ayude a ir comprendiendo la narración que el maestro realiza. Indica el autor la pertinencia de esta forma en la enseñanza de la religión combinada con la Historia Sagrada, pero además resalta que en algunas ocasiones debe acudirse a la pintura para poder acceder a los objetos que se relacionan con ésta. La enseñanza se hace objetivas cuando podemos acceder a los objetos directamente y se objetiviza (sic) cuando lo hacemos a través de la pintura (Zamora, I924: II3).

\section{Los procedimientos pedagógicos}

Ahora, en cuanto a los procedimientos pedagógicos que en la época Zamora relaciona y que considera tan importantes que algunos son «conocidos como métodos», se definen como «el modo u orden que se sigue en la enseñanza de las ciencias para hallar la verdad» (Zamora, I924: II4).

El primer procedimiento es el Objetivo, conocido también como concreto. En este procedimiento se parte de lo que el niño conoce para llegar a lo desconocido, por lo cual, está enlazado con el método de evidencia previa. Al mostrarle al niño un objeto que ya conoce y hacerle preguntas sobre éste por lo general surgen ideas nuevas y se enriquece su entendimiento.

El segundo procedimiento es denominado Subjetivo, entendido por nuestro autor como "aquél en que se hace caso omiso del objeto» y en algunos momentos hay quienes lo llaman abstracto (Zamora, I924: II5). Este procedimiento a diferencia del anterior se centra en la imagen del objeto y no en el objeto mismo, lo que a pesar de ser subjetivo, también, produce efectos positivos en los niños. La diferencia que caracteriza realmente a estos dos procedimientos la encontramos en lo ocurrido con la escalera de Pestalozzi, según Zamora, ejemplo que retoman Sáenz y otros (1997) de Johannes (I887):

Cuando el pequeño cantón suizo de Niedwalden fue desvastado por los franceses a fines del xviII, más de cien niños de las aldeas destruidas fueron reunidos en un antiguo convento de Stanz, y Pestalozzi se encargó de ellos [...] Careciendo de textos, sus enseñanzas tuvieron que ser orales, y para las explicaciones se valía de láminas y de todo lo que interesaba la curiosidad de los niños [...] Se dice que [...] un día hablaba de una escalera portátil, pero la lámina que la representaba se había extraviado, y entonces un niño, al notar las perplejidades del maestro, le indicó que cerca de la puerta había una escalera que tal vez podría servirle, a falta de la lámina. Esto fue un rayo de luz. La sugestión del niño hizo que se le ocurriera a Pestalozzi usar los objetos mismos en

s Alude aquí a la enseñanza objetiva muy en boga a partir de los planteamientos de Pestalozzi. 
la enseñanza, como mucho más a propósito que sus representaciones, y he aquí cómo tuvo principio la enseñanza objetiva (Johannes, I887, citado en Sáenz, Saldarriaga y Ospina, 1997).

El tercer procedimiento, llamado Abstracto, «es aquél en que propiamente hay abstracción del objeto material o de su imagen representada, y puede confundirse con el procedimiento oral, aunque en este último caso, dada la habilidad pedagógica del educador, puede hacerse tan interesante la enseñanza que casi es concreta...» (Zamora, I924: II5), este procedimiento es aplicado generalmente a la enseñanza de la aritmética y de las ciencias naturales.

El cuarto procedimiento descrito es el Analítico, y podemos comprenderlo de la siguiente manera: si deseamos enseñar algo por ejemplo una letra ${ }^{6}$ podemos apoyarnos en un objeto cuya pronunciación comience o termine con dicha letra, luego al dividir la palabra en sus elementos necesariamente se llegará al sonido y se habrá empleado un procedimiento analítico, se define entonces, como «aquél en que se va del todo a las partes, de lo general a lo particular, tomando los elementos para hacer comprender mejor la materia y obtener fruto satisfactorio» (Zamora, I924: II6).

El quinto procedimiento lo identifica como Sintético y es contrario al anterior, aunque se apoyan mutuamente formando uno mixto que recibe el nombre de sintético-analítico o analítico-sintético. El autor lo define como «aquél en que se condensa la enseñanza, pues se va de las partes al todo, de lo particular a lo general, de lo simple a lo compuesto» (Zamora, I924: II6). Era utilizado en la época para la enseñanza del dibujo y su aplicación en la primaria era bastante extendida.

El sexto procedimiento, llamado Oral, consiste principalmente en expresar las ideas que se tienen en forma oral o como dijera Zamora de «viva voz». Este procedimiento puede utilizarse para todos los niveles educativos, y es el más utilizado en conferencias, seminarios, congresos y otros eventos que reúnen gran cantidad de personas. Subraya como importante que quien utilice este procedimiento tenga en cuenta el público al cual va dirigida su intervención y se debe adiestrar con tesón y dedicación en su empleo.

El séptimo es el procedimiento Inductivo que consiste en «el orden que seguimos al dictar una clase, para buscar una verdad» (Zamora, I924: II7). Tal procedimiento tiene gran importancia, pues el maestro se convierte en un guía para el educando conduciéndolo de tal manera que éste realice un trabajo mental eficiente que le ayude a adquirir y atesorar conocimientos y es tal su importancia que algunos lo llaman método. Al llevar a la práctica este procedimiento, debe tenerse el cuidado suficiente como para no dejar en el educando dudas, por esto, este procedimiento se relaciona muy de cerca con la forma interrogativa que le sirve como una herramienta para lograr mejores resultados.

El octavo procedimiento es el Deductivo, que debe ser la pareja inseparable del anterior. La deducción es el efecto y la inducción la causa. Si esta última es bien manejada por el maestro se presume que el niño deducirá correctamente ya que una buena pregunta derivará probablemente en una buena respuesta.

El noveno procedimiento es el Memorial, que tiene como base el entendimiento, pues sin éste no es racional (el procedimiento) ya que su principal objetivo es cultivar la memoria. Es aplicable cuando se trata de principios, leyes especiales y en asuntos relacionados con la enseñanza de la fe.

6 Sin duda, Zamora se refiere a un fonema. 
El décimo, conocido como procedimiento Experimental, se fundamenta en la experiencia y ha arrojado muy buenos frutos a través de los años. Actualmente, hay áreas en las que no sólo es aplicable sino necesario, como por ejemplo en las matemáticas, la física, la biología, por mencionar sólo algunas.

El undécimo procedimiento es el Mecánico, y tiene como finalidad el aprendizaje textual de lo que el maestro desea que el niño sepa, sin importar si éste lo comprende. Es un procedimiento bastante criticado y que no aporta al desarrollo intelectual del niño, pues es muy probable que no sepa expresar nada con sus propias ideas. Es utilizado generalmente por falsos pedagogos que creen que cuando el niño repite una lección, impuesta por ellos, ha logrado un real aprendizaje. Muchos padres de familia, el niño e incluso el mismo maestro son engañados así como la sociedad en general. A este respecto Zamora cita una célebre frase de Pestalozzi que dice: "Yo no quiero que mis discípulos sepan sino aquello que pueda serles demostrado, como dos y dos son cuatro» (Zamora, I924: II8). Éste llega a ser aceptable sólo con el concurso de algunas formas y otros procedimientos y para el inicio del educando en la lectura.

Gráfico original en el que Zamora sintetiza los procedimientos pedagógicos

(ZAMORA, I924: II9)

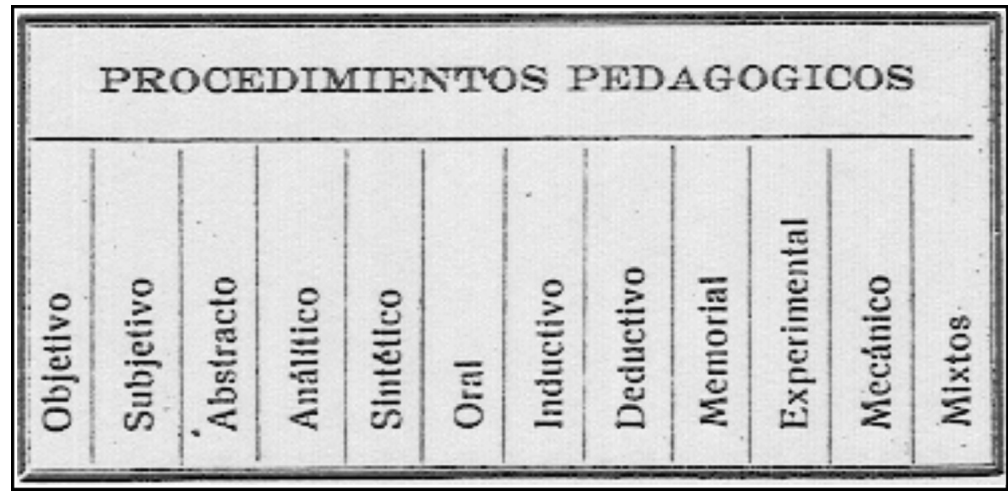

\section{Los sistemas pedagógicos}

En cuanto a los sistemas pedagógicos, Zamora los define como «el conjunto de reglas o principios de la enseñanza, enlazados entre sí», y los clasifica en el Mutuo o Recíproco, el de Conferencias y el de Discusión (Zamora, I924: II9).

El sistema Mutuo o recíproco es entendido por nuestro autor como «aquel que, mediante un intercambio de ideas, conduce a hacer luz, a buscar una verdad...» (Zamora, I924: II9). Sugiere la utilización de este sistema para personas de cierta edad y con cierto tipo de conocimientos. Sin embargo, el sistema Recíproco puede ser utilizado por aquellos estudiantes aventajados de la escuela primaria, con el inconveniente de no contar con la vigilancia del maestro y basarse solo en la repetición que un educando puede lograr que realicen sus compañeros de una determinada lección, lo que, como es obvio, traía consigo la indisciplina que en la época era rechazada totalmente. 
En segunda instancia tenemos el sistema de Conferencias, considerado en la época como uno de los mejores y empleado por los países más adelantados en materia educativa como Alemania, Inglaterra, Francia, Suiza y otros países. Sin embargo, no se consideraba adecuado para la escuela primaria; era ideal para la escuela superior donde el educando podía hacer sus propios resúmenes y tomar nota de los aspectos que más le interesaban. Hoy sabemos que este sistema es ampliamente utilizado a nivel mundial.

El tercer y último sistema, llamado de Discusión, no era muy aceptado en la época para ningún nivel, pues escuchar las ideas o dudas de los educandos y crear algún tipo de discusión alrededor de esto, consideraba Zamora, era una forma de agotar el tiempo de la clase y hacer que ésta terminara rápidamente.

Gráfico original en el que Zamora sintetiza los sistemas pedagógicos

(ZAMORA, 1924: I2I)

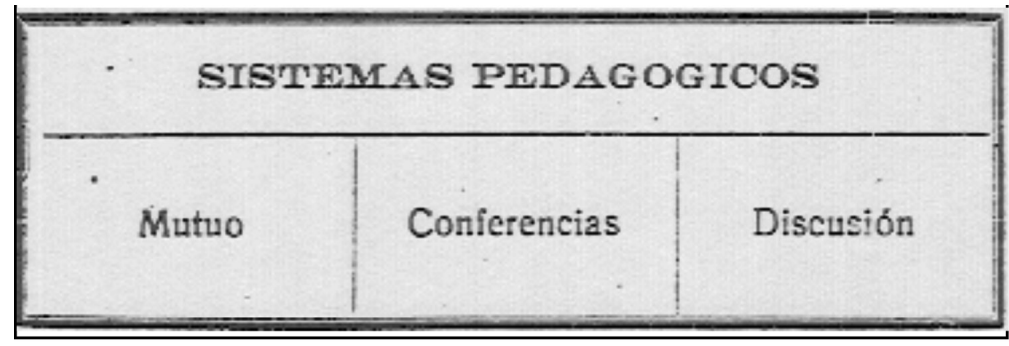

\section{Las leyes pedagógicas}

Finalmente Zamora trata las leyes pedagógicas, que le son esenciales y se relacionan directamente con la enseñanza, enmarcadas dentro de la clasificación que ha realizado de la educación: la educación física, la intelectual y la moral.

Las leyes de la educación física-que las divide en seis- tratan sobre higiene y filosofía, los alimentos, el calor y el aire, la luz, el ejercicio y el descanso, siendo algunas de éstas que la especie, calidad y cantidad de alimentos que se suministren al niño estén de acuerdo con su naturaleza. Que la luz sea suficiente, distribuida con habilidad y dirigida convenientemente, proporcionándola por el lado izquierdo para los trabajos escolares. Que la posición en los ejercicios escolares debe obedecer a la comodidad y a la variación, ejercitando a diario la actividad muscular... Tales leyes, que están inspiradas en los modelos europeos y norteamericanos vigentes, desconocen la realidad de la educación que se impartía en el país, pues en los informes de los supervisores de la época, una queja constante es que las escuelas están pobremente iluminadas, que los escolares van a la escuela sin haber ingerido los alimentos necesarios y que la dotación de bancas para el estudio no siempre alcanza para todos (Zamora, 1924: I2I).

De la misma manera, resume las leyes de la educación intelectual, entre las cuales propone que se empiece la enseñanza por la educación de los sentidos, mediante las lecciones objetivas. Que la observación sea siempre la base cierta del aprendizaje de una regla o de un principio, pero a medida que el desarrollo mental evolucione se procure el menor uso posible de los sentidos externos. Que el maestro se prepare

7 Zamora las cuantifica en nueve leyes. 
convenientemente para dar la enseñanza... Aquí, la influencia pestalozziana es muy clara, y son claves las alusiones a la observación previa, a la educación de los sentidos y a la preponderancia que se le da al desarrollo mental en consonancia con lo que ocurría en el entorno del niño al sugerir aspectos como tener en cuenta la evolución de la mente del niño, que la enseñanza esté basada en la evidencia y la lógica, si se percibe cansancio el maestro debe suspender la enseñanza (Zamora, I924: I23). Si todo esto se aplica correctamente, el niño debe quedar preparado para dirigirse por sí mismo, lo que hoy conocemos como el aprendizaje autónomo planteado por Ausubel.

Finalmente, establece como leyes para la educación moral que el maestro conozca el orden moral, y esté convencido de la verdad y sus preceptos. Que la voluntad del niño obedezca a los impulsos del entendimiento para que sus hábitos puedan perfeccionarse. Que el maestro siempre conserve la autoridad y su carácter de superior acostumbrando al niño a obedecer sin imposición. Meditar la orden que se dé para que no sea necesario repetirla. Enseñar la caridad a través de ejemplos. No castigar frecuentemente ya que esto crea animadversión. Preparar a los niños en los bancos de la escuela para gobernarse a sí mismos (Zamora, I924: I24).

\section{Conclusiones}

Sin duda el recorrido hecho a través de este texto evidencia que los aportes y la claridad con que Zamora aborda la educación en sus diferentes aspectos físicos, intelectual y moral eran avanzados y muy actuales a su época, haciendo uso de ideales que circulaban en el mundo de los grandes pedagogos del momento, muchos de estos aportes se encuentran hoy en día más vigentes que nunca, aunque mucha veces con nombres diferentes, pero si escudriñamos un poco vemos que realmente se trata de lo mismo o muy similar, no en vano se observa la gran influencia de Pestalozzi, uno de los precursores más importantes del constructivismo. Obviamente es de resaltar que en la época se hacía mucho más énfasis en la educación moral, un valor en la educación que comenzamos a extrañar.

Además, la forma como el autor trata los diferentes métodos, procedimientos, formas, sistemas y leyes de la educación permiten al docente e intelectuales interesados en el tema hacerse con unos conceptos claros y precisos para luego introducirse en la gran complejidad que estos temas han adquirido hoy en día. En definitiva, el poder aunar todos estos elementos para poder lograr un buen proceso de enseñanzaaprendizaje es un arte.

\section{Bibliografía}

Flórez, R. (1994) Hacia una Pedagogía del Conocimiento. Santafé de Bogotá: McGraw-Hill. Johonnot, J. (1997) Principios y Práctica de la Enseñanza. Nueva York: Appleton y Cía., I887, p. II2; citado por Restrepo Mejía, Martín y Luis. Elementos de la pedagogía. Obra adoptada como texto para las escuelas normales de Colombia y recomendada para la enseñanza de la materia en el Ecuador. Bogotá: Imprenta Moderna, I9II, p. I72, 2 vols. En SÁENZ, Javier; Saldarriaga, Óscar y Ospina, Armando. Mirar la Infancia: pedagogía, moral y modernidad en Colombia, 1903-1946, vol. I. Colciencias-Foro Nacional por Colombia-UniandesUniversidad de Antioquía-Clío. Medellín.

Zamora, J. M. (1924) Cursos de Historia, Metodología General y Especial y Legislación de la Instrucción Pública. Pedagogía. Bogotá: Tipografía Augusta. 